\title{
Complexity and Dynamic Modeling of Urban System
}

\author{
Wensheng Zhou and Qiang Li
}

\begin{abstract}
Complex system theory has made people recognize and understand urban phenomena from a new perspective. In particular, The research method of the complex system has provided a powerful tool to explore problems of urban system. In this paper, basic theories of research on complexity science and complex system are described, the necessity of dynamic modeling and simulation methods as well as general steps are analyzed for the complex system. Based on this, the complexity of urban system is discussed and analyzed. Besides, the means by which dynamic simulation methods of complex system are applied in studying urban system and their significances are discussed. The result shows that it is necessary to depict, analyze and know about problems on urban complexity and explore deeper rules hidden in dynamical urban development with the theoretical framework of complexity science, so as to better interpret, design and plan cities.
\end{abstract}

Index Terms-Complexity science, CA, MAS, urban system.

\section{INTRODUCTION}

With the rapid development of cities and constant integration of disciplines, urban planning has increasingly become a discipline not purely focusing on designing physical forms, but has also begun to pay great attention to other non-physical factors (e.g. human and society) and more fundamental rules hidden in dynamic development of cities. Studied by urban planners, urban system is becoming more and more complex in both forms and nature. Thus, how to understand, analyze and depict such a complex system has become an urgent problem to be solved by planners. Traditional research methods based on reductive thinking encounter great challenges in interpreting the formation of urban structure, urban spatial evolution and laws of human activities in cities, etc, which fail to reveal deeper causes and rules of dynamic urban development.

By the end of 1980s, the founding of Santa Fe Institute directly facilitated and led to the emergence of complexity science. The complexity science had achieved rapid development within less than 20 years and has become the focus of attention in the scientific field at present. On one hand, the theories of complexity science greatly change people's ideas of urban system. On the other hand, research methods of complex system particularly make powerful means available to explore urban system. The rest of the paper is organized as follows: The basic theories of research on complexity science and complex system are described in

Manuscript received September 6, 2013; revised October 17, 2013. This work was supported by the National Science Foundation of China (No. 51178236).

Zhou Wensheng is with School of Architecture, Tsinghua University, Beijing, 100084, China (e-mail: zwsbj@163.com).

Li Qiang is with 295847 Department of PLA, Beijing, 100120, China (e-mail: liquider@sina.com).
Section II. Section III addresses the complexity of urban system. Section III discusses the modeling and simulation of complex systems and modeling of urban system. The paper is concluded in Section V.

\section{MAIN THEORIES OF COMPLEXITY SCIENCE}

The exact connotation and extent of complexity science remain to be discussed [1], [2], while its theoretical system has never been clearly defined by now. Generally, the main research object of complexity science is the complexity of a complex system. In essence, complexity science is supposed to be the continuation and deepening of system movement, so theories of "system" oriented modern systems science and nonlinear science shall be the major sources from which theories of complexity science originate. Meanwhile, complexity science has some of its own unique theories such as theories of complex adaptive systems and artificial life. The framework of complex systems theory is shown in Fig. 1, which expresses the relationships of complex systems theory with system science and nonlinear science as well as the development process and status of complex systems theory.

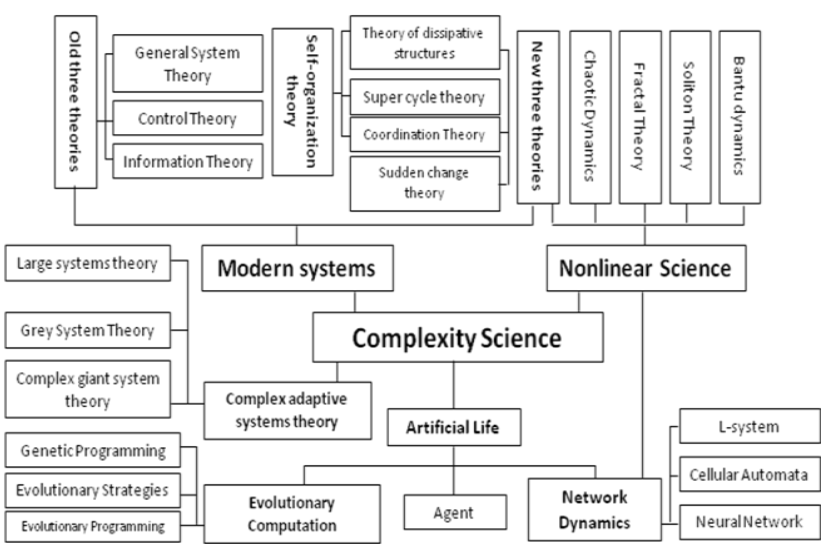

Fig. 1. Theoretical framework of complex systems [3].

Although there are a variety of theories and no consensus has been fully reached on complexity and complex systems, some important conclusions have been approved by the scientific circle. Complex systems exist objectively and they are not determined by human knowledge. There are nonlinear interactions among microscopic individual components of complex systems and it is impossible to deduce all overall characteristics according to partial attributes. Complex systems are definitely dynamic, some novel characteristics of which spontaneously come into being during evolution. Complex systems aren't intuitively presented and openness is the fundamental condition for complex systems. Adaptivity is the major cause of complexity, while nonlinearity and emergence are important features of complex systems. On the whole, complex systems theory is still a theoretical system 
remaining to be constantly improved and expanded by the concerns and efforts of scientists from different fields of all countries.

\section{COMPLEXITY OF URBAN SYSTEM}

The research methods of complex systems theory and complex systems have made some crucial concepts and methodologies available for us to study urban problems. Cities are expressed as social carriers in which materials are greatly accumulated. Many elements such as social organizations, people flow, material flow and accumulation of historical culture, etc are assembled, blended, fermented and synthesized in such giant material carriers, for which new materials, energy, information and social elements are produced [4], so a city can be regarded as a complex giant system.

Many scholars have discussed the complexity of cities and urban system from different perspectives [5]-[7]. We can know about the complexity of an urban system from following aspects.

\section{A. Multiple Elements of Urban System}

All complex systems are composed of multiple elements which have different characteristics, structures and functions. An urban system is just such a structure made up of several elements. The elements constituting cities contain material elements (including air, sunshine, soil, water, building and organisms) and nonmaterial elements such as belief, religion, thought, ideology, economy and society. There are not only a great variety of such elements, but also various connections among these elements and their sub-elements. Thus, different kinds of coupling mechanisms form, all of which constitute a huge system like a city.

\section{B. Multiple-Layer Structure of Urban System}

Multiple elements composing the system are not key factors leading to the complexity of a complex system, whereas the multi-layer structure presented when the elements constitute the system is the key for complexity. The more layers a system has, the higher the complexity. A city is composed of multiple layers including people, families, neighborhoods, streets, communities, districts and cities which constitute several sub-systems. Finally, a large system (city) is made up of these sub-systems, the layers of which are intricate and complex with close connections. A city is a huge $\mathrm{N}$-dimensional system if each sub-system, layer and connection in the city are treated as one of its dimensions.

\section{Openness of Urban System}

According to non-equilibrium self-organization theory, openness is the essential condition for the system to transform from a disorderly state to an orderly state. Urban space is a typical open system with dissipative structure, which shall maintain extensive connections with the outsides, constantly introduce negative entropy flow (e.g. personnel, materials, energy, information and capital, etc) and keep on outputting positive entropy flow such as products and waste. Only in this way can its structure keep stable and orderly. The openness of system will inevitably result in an increase of factors affecting system movement and the increase in variables about system movement will objectively cause the complexity of system. In a word, openness and dissipativeness are not only inherent characteristics of an urban system, but also basic conditions for the formation of a complex urban system.

\section{Dynamic Inner Conditions of Urban System}

People are the most fundamental elements of a city in which human beings can study, accumulate knowledge and experience through living, who also actively and adaptively change their own behaviors. On the premise that all individuals are active and adaptive, the entire urban system develops its learning and adaptive behaviors. CAS theory considers that adaptivity is the major cause of complexity, while the adaptivity of an urban system leads to dynamic changes of spatial scope, population size, economic development level and social class with time in other regional spaces. Such changes promote the transfer of a city between orderly and disorderly states.

\section{E. Nonlinear Interactions among All Elements and Sub-Systems of an Urban System}

Urban system is a typically complex nonlinear dynamic system. There are common nonlinear interactions between all elements and sub-systems, which include nonlinear evolution of a single element or a sub-system of a city (e.g. the urban population can never achieve infinite growth), nonlinear interactions between urban elements (e.g. nonlinear allometric growth relationships between urban population and area) and nonlinear interactions among urban sub-systems (e.g. double-lognormal distribution of urban population, area and product) [8]. Nonlinearity is the core feature of an urban system and one of the fundamental causes of complexity of an urban system.

\section{F. Self-Organizing Evolution of Urban System Structure and Orders}

Self-organization is a basic characteristic of open complex systems such as life system and social system. As the founder of synergetics, Haken gave a classical definition to "self-organization" that a system shall be self-organizing if it isn't specifically interrupted by external factors in the process of gaining spatial, temporal or functional structures and its structure and functions are the results of its inner contradictions but not imposed by external factors [9]. The evolution of urban system structure and orders has remarkable self-organizing features as well. Urban self-organizing mechanism is coupled by self-growing, self-replicating, self-isolating and self-optimizing mechanisms. Fang Yangang deems that urban system didn't have a predetermined macroscopic objective and structure from the very beginning, and it is a self-organizing complex spatial system formed due to interactions of microscopic urban subjects under certain constraints and limited rational guidance for maximizing self-efficacy (survival, safety, respect and self-actualization) [10].

Hence, a city can be said to be a open complex giant system and a human-centered organic whole of extremely close connections with the society based on economic activities under the natural environment, which has complicated characteristics such as multiple elements, 
multiple layers, openness, dynamic characteristics, nonlinearity and self-organization.

At present, the research on complexity of an urban system has attracted widespread interests of architects, planners and urban geographers, which means studying complex components of an urban system in different layers, understanding the mechanisms of interactions (among all elements, subsystems, between systems and environment), exploring macroscopic orders (structure, function, behavior and evolution, etc) incurred by microscopic mechanisms, examining the control and synergy of macroscopic orders for microscopic elements, and studying dynamics, rules, process and modes of the evolution of an urban system, for the purpose of providing new ideas and methods for urban planning.

\section{Modeling OF URBAn SyStem}

\section{A. Modeling and Simulation of Complex Systems}

Modeling is an effective experimental technique for human to learn about the world and human's exploration of world is a process for establishing various models. A model is a simplified depiction of cognitive objects and a specific pattern formed by modeling prototype [11]. It is a substitute for a real system, by which less time and cost are spend in experiments. The model can be repetitively demonstrated and studied for observing the behaviors of systems more easily.

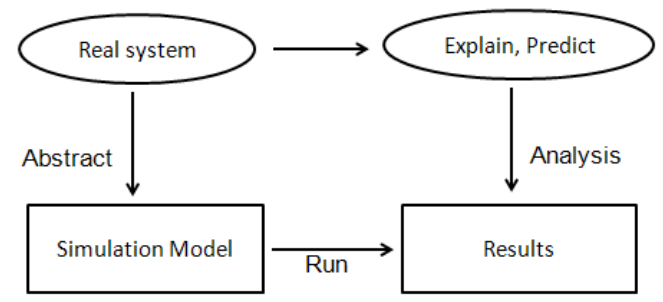

Fig. 2. Relationships between simulation models and real systems [12].

With the development of science and technology, the theories for depicting things by models have become increasingly perfect and research has been conducted more and more extensively. Meanwhile, modeling techniques are constantly developing and new models are continuously emerging. Generally, models can be divided into physical, qualitative and quantitative models according to forms [12].

Computer model, also known as computational simulation, is a special qualitative model, by which the running process of a real system is reproduced by computer simulation to solve solutions for the characteristics of a real system. Fig. 2 shows the principles and process of computer simulation. First of all, a simulation model of the real system is established pursuant to principles of similarity, by running which simulation results are obtained. After analyzing the simulation results, essential laws of the real system are gained to interpret and forecast the system. Compared with the experiments on real systems and traditional modeling, computer modeling has plenty of advantages. For instance, it is economical, safe, reliable, flexible and predictable, which can be repetitively used for multiple times. By computer modeling, many systems which can't be directly tested can be studied by simulation experiments to solve lots of complex problems which can't be described or analyzed by mathematical means. It is because of these unique advantages that computer simulation becomes an important method for present scientific research. It is paid more and more universal attention and honored as the "Third Method for Scientific Research".

It is rather difficult to reproduce complex economic, social, biological and ecological systems because they are impacted by too many uncontrollable factors, so complex systems are essentially non-repeatable systems. Besides, their open, emergent, nonlinear, dynamic properties and complex correlations make mathematical analysis unavailable to them, thereby these systems are also non-computable. For the research on non-repeatable systems especially non-computable systems, simulation is "an important and even the sole research method" [13]. It is impossible for researchers to solve the characteristics of a real system unless the running process of a real system is simulated and reproduced on a computer by building a simulation model. Complex systems can't be studied without computer models and typical models of complexity science like traditional natural science can't exist without laboratories. As modern hardware and software technologies develop and become mature, requisite conditions are available to the modeling and simulation of various systems due to the arithmetic capabilities provided by these technologies, in order that huge complex systems can be simulated by computer modeling. Therefore, computer simulation is said to be a crucial approach to studying complex systems and even the sole means for examining many systems.

\section{B. Modeling of Urban System}

As urban complexity is understood and studied, modeling and simulation of urban system gradually becomes important research methods in the disciplinary fields of urban planning, urban economics and urban geography, etc as creative research ideas.

At present, complex systems can be modeled and simulated in plenty of ways including system dynamics modeling, microscopic simulation modeling, cellular automata modeling, neural network modeling, genetic algorithm modeling and multi-agent system, etc, among which cellular automata (CA) and multi-agent system (MAS) are major approaches to model urban system since they correspond to urban simulation.

CA is a parallel mathematical model of discreet time-space and state with self-organized behaviors [14], which is commonly used in the research on complex systems. Although its microscopic rules are relatively simple, it may have rather complex behaviors. In the research fields of physics, chemistry and biology, the reproduction, self-organization and evolution of complex systems have been successfully simulated. As a dynamical model of spatial-temporal features, CA isn't only utilized to simulate and analyze ordinary complex systems, but also has great advantages in simulating urban system. Tobler, from University of Michigan, formally simulated the development of Detroit of Great Lakes for the first time in 1970s [15]. Thereafter, Helen Couclelis [16], Batty [17], Clarke [18], Li 
and Yeh [19] successively studied geographical and urban cellular automata, who made a good many meaningful achievements.

Chinese scholars have gained plenty of outstanding achievements in the research on cellular automata and urban system too. Zhou Chenghu et al. studied urban spatial development and evolution by CA, who constructed GeoCA-Urban model and proposed the concept of GeoCA (geographical cellular automata) [20]. Li Xia and Ye Jia' an et al. studied the impacts of global, regional and local constraints on the simulation of cellular automata, who simulated the forms of sustainable urban development by constrained cellular automata [21] and examined the methods by which cellular automata is used for urban planning [22]. In addition, He Chunyang, Shi Peijun [23] et al. studied the evolution of urban land uses and urban development process by CA.

Theory and technology of MAS have developed based on the theory of complex adaptive systems, artificial life and technology of distributed artificial intelligence (DAI), which are ideological methods and tools for analyzing and simulating complex systems. According to the research findings in the field of distributed artificial intelligence, models are established for complex systems by bottom-up thoughts. Microscopic individual components in a model may have rich attributes, nearly real decision-making logics and behavioral characteristics. With the interactions among individual components and their interactions with environment, macroscopic features of a system emerge to build a bridge between micro and macro.

MAS can produce a global macroscopic pattern through the interactions among microscopic individual components to better understand phenomena and discover hidden mechanisms, so it may play unique roles in studying urban problems such as urban spatial evolution, population migration, traffic control, public safety emergencies, environmental resources management, public facility location and sustainable development, which provides a brand new perspective for studying urban problems. Thomas Schelling put forward the first MAS-based urban segregation model in 1971 [24]. The "city" simulated with chessboard has gone through a self-organizing process of microscopic interactions, wich has fully reflected the principle of "producing orders from instabilities". In 1998, Benenson simulated self-organizing phenomena of urban spatial evolution, segregation of urban residents and residential division by multi-agent models [25]. In 2001, Ligtenberg proposed a land use planning model based on cellular automata and multi-agent, while governmental planning factors are introduced into this model [26]. In 2003, Brown simulated the development of residential districts in outlying suburbs by a multi-agent model [27]. In China, Xue Ling (2003) designed an urban evolution model by multi-agent modeling under the Swarm environment [28], to explore the evolution of macroscopic spatial structures resulted from the interactions of microscopic agents such as urban residents and enterprises. Yang Qingsheng and Lixia et al. (2005) examined urban land use changes by multi-agent model and CA [29].

It can be seen from aforementioned studies that CA model is more suitable for simulating urban environmental elements such as urban land uses and facilities, while MAS is more adaptable to simulate behavior subjects such as people, vehicles and organizations in cities for their distinctive characteristics.

With the application of dynamic modeling and simulation methods such as CA and MAS in studying urban system, effective tools have been provided for people to understand, analyze and depict urban system and studying urban phenomena by experiments have become possible. Traditionally, natural science can be studied by experimental methods, whereas such methods are applicable to social science. Urban science is a category of social science to a great extent, because the existence of a city requires social, economic and cultural support systems beyond material phenomena. Essential urban rules just exist in these nonmaterial patterns. Due to unique theoretical perspectives of complexity science, an artificial society or a virtual world can be built by constructing a model for microscopic individual components and letting these components have complex interactions through dynamic modeling and simulation of complex systems, so as to map real urban societies on the computer and observe the macroscopic mode presented by the system to discover general rules hidden in phenomena by repetitively running the artificial society on the computer. Hence, the modeling of urban system makes it possible to study real urban system which can't be experimentally tested by models. In this case, it gets possible to study urban system by experiments.

\section{CONCLUSIONS}

Analyzing and solving problems by thoughts of complexity science is a considerable progress made by human philosophical thinking. A city is a open and complex geospatial system, which has typical features of complex systems. Due to the complexity of an urban system, theoretical methods of the complex system should be applied in studying problems on cities. It is necessary to depict, analyze and know about problems on urban complexity and explore deeper rules hidden in dynamical urban development with the theoretical framework of complexity science, so as to better interpret, design and plan cities.

\section{REFERENCES}

[1] H. S. Wei, Complexity Research and Systematic Thinking Approach, Beijing: People Press, 2007, pp. 84-196.

[2] B. L. Hao. "Characterization of complexity and 'The Science of Complexity'," Science, vol. 51, no. 3, pp. 3-8.

[3] S. Y. Liao, "Research on agent-based modeling and simulation of complex system," Ph.D. dissertation, Graduate School of National University of Defense Technology, Changsha, China, 2005.

[4] H. M. Duan and D. W. Yang, "Preliminary study on mathematical description of the complexity of urban system," Human Geography, vol. 10, no. 3, pp. 112-115, 2007

[5] L. Y. Wu, An Introduction to the Sciences of Human Settlements, Beijing: China Building Industry Press, 2001.

[6] G. Z. Zhou, "City and its region: An typical open complex huge system," Urban Planning, vol. 26, no. 2, pp. 7-8, 2002

[7] T. Zhang, "Studies on the promotion of complex considerations," Complexity Research, Committee of Chinese Academy of Science, Ed. Beijing: Science Press, 1993, pp. 1-6.

[8] Y. G. Chen and J. Luo, "A dynamic similarity analysis of fractal structure of Zhengzhou City - empirical study of the relationships 
between urban population, lands and output," Economic Geography, vol. 21 , no. 4 , pp. 389-393, 2001

[9] H. Haken, Synergetics, translated by F. H. Ling, Shanghai: Shanghai Translation Publishing House, 2001.

[10] Y. G. Fang, "A study on the complexity of urban geographical and spatial system," Ph.D. dissertation, Northeast Normal University, Changchun, China, 2006.

[11] M. Q. Fang and S. R. Zhang, Modeling and Simulation of Complex System, Beijing: China Renmin University Press, 2005.

[12] H. Y. Xuan and F. Zhang, Simulation and Application of Complex System, Beijing: Tsinghua University Press, 2008, pp. 3-4.

[13] H. L. Li, "The agent-based distributed simulation for complex Systems," Ph.D. dissertation, Graduate School of National University of Defense Technology, Changsha, China, 2001.

[14] C. H. Zhou, Z. H. Sun, and Y. C. Xie, The Research of Geographical Cellular Automata, Beijing: Science Press, 2001.

[15] W. R. Tobler, "Cellular geography," Philosophy in Geography, pp. 279-386, 1997

[16] H. Couclelis, "Of mice and men: what modent populations can teach us about comples spatial dynamics," Evoironment and Planning A, vol. 20 pp. 99-109, 1988.

[17] M. Batty and Y. Xie, "From cells to cities," Environment and Planning $B$, vol. 21, pp. 531-548, 1994.

[18] K. C. Clarke, S. Hoppen, and L. Gaylos, "A self-modifying cellular automaton model of historical urbanization in the San Francisco Bay area," Environment and Planning B, vol. 24, pp. 247-261, 1997.

[19] X. Li and A. G. Yeh, "Modeling sustainable urban development by the integration of constrained cellular automata and GIS," International Journal of Geographical Information Science, vol. 14, no. 2, pp. $131-152,2000$

[20] C. H. Zhou, Z. L. Sun, and Y. C. Xie, The Research of Geographical Cellular Automata, Beijing: Science Press, 2001.

[21] X. Li and J. A. Ye, "Constrained cellular automata for modelling sustainable urban forms," Journal of Geographical Science, vol. 54, no 4, pp. 289-298, 1999

[22] X. Li, J. A. Ye, and X. P. Liu, "Geographical simulation systems for urban planning," Urban Planning, vol. 30, no. 6, pp. 69-74, 2006.

[23] C. Y. He, P. J. Shi, J. Chen, et al., "A study of land use scenarios dynamics model based on system dynamics model and cellular automata," Chinese Science (D), vol. 35, no. 5, pp. 464-473, 2005

[24] T. C. Schelling, "Dynamic models of segregation," Journal of Mathematical Sociology, no. 1, pp. 143-186, 1971.

[25] I. Benenson, I. Omer, and E. Hatna, "Entity-based modeling of urban residential dynamics: the case of Yaffo, Tel Aviv," Environment and Planning B, no. 29, pp. 491-512, 2002.
[26] A. Ligtenberg, A. K. Bregt, and R. V. Lammeren, "Multi-actor-based land use modeling: spatial planning using agents," Landscape and Urban Planning, no. 56, pp. 21-33, 2001.

[27] D. G. Brown, S. E. Page, R. Riolo, and W. R, "Agent-based and analytical modeling to evaluate the effectivess of greenbelts," Environment Modeling \& Sofeware, vol. 19, no. 12, pp. 1097-1109, 2003.

[28] L. Xue and K. Z. Yang, "Research on urban evolution using agent-based simulation," Systems Engineering Theory and Practice, vol. 12, pp. 1-9, 2003

[29] Q. S. Yang, X. Li, and X. P. Liu, "Urban land use change research based on agent and cellular automata," Geo-Information Science, vol. 7, no. 2 , pp. $78-81,2005$.

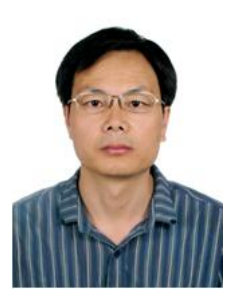

Wensheng Zhou was born in 1966 in Lanzhou, China. He got the B.S. degree and M.S. degree in Engineering Surveying in 1987 and 1999 from Wuhan Technical University of Surveying and Mapping, Wuhan, China, and $\mathrm{Ph} . \mathrm{D}$. degree in Mapping and Geographic Information Engineering in 2003 from Wuhan University, Wuhan, China. He is presently an associate professor of the School of Architecture, Tsinghua University, Beijing, China. His main fields of research are geodesign and geographical simulation, smart city and the application of spatial information technique in cultural heritage protection. He has authored and coauthored more than 60 papers in Journals and Conferences and has 2 books on GIS, urban planning and cultural heritage protection.

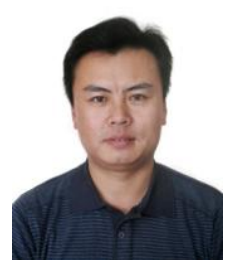

Qiang Li was born in Zhaoyuan, Heilongiiang Province, China in 1973. He got the bachelor of Engineering degree in Computer Software in 1996, the master of Engineering degree in Computer Application in 2002 from The PLA Information Engineering University, and the Ph.D. degree in Architecture from Tsinghua University in 2010. Now he is a Senior Engineer of PLA 95806 Unit. His research interests lies around Agent Based Modeling (ABM) with specific interest in urban system dynamic modeling, combat simulation and war-game. 